\title{
On the Conjectures Regarding the 4-Point Atiyah Determinant
}

\author{
Mazen N. BOU KHUZAM ${ }^{\dagger}$ and Michael J. JOHNSON ${ }^{\ddagger}$ \\ $\dagger$ American University of Iraq, Suleimaniya, Street 10, Quarter 410, \\ Ablakh area Building no. 7 Sul, Iraq \\ E-mail: mazen.boukhuzam@auis.edu.iq \\ $\ddagger$ Department of Mathematics, Faculty of Science, Kuwait University, Kuwait \\ E-mail: yohnson1963@hotmail.com
}

Received January 15, 2014, in final form June 23, 2014; Published online July 05, 2014 http://dx.doi.org/10.3842/SIGMA.2014.070

\begin{abstract}
For the case of 4 points in Euclidean space, we present a computer aided proof of Conjectures II and III made by Atiyah and Sutcliffe regarding Atiyah's determinant along with an elegant factorization of the square of the imaginary part of Atiyah's determinant.
\end{abstract}

Key words: Atiyah determinant; Atiyah-Sutcliffe conjectures

2010 Mathematics Subject Classification: 51K05; 51P99

\section{Introduction}

The Atiyah determinant is a complex-valued determinant function $\operatorname{At}\left(P_{1}, \ldots, P_{n}\right)$ associated with $n$ distinct points $P_{1}, \ldots, P_{n}$ of $\mathbb{R}^{3}$. It was constructed by M.F. Atiyah in [1] in his attempt at answering a natural geometric question posed in [3] and arising from the study of the spin statistics theorem using classical quantum theory. The original conjecture of Atiyah was that At does not vanish for all configurations of distinct points $P_{1}, \ldots, P_{n} \in \mathbb{R}^{3}$. The conjecture was verified in the linear case (all points lie on a straight line) and in the case $n=3$ by Atiyah in [1]. However, the case $n \geq 4$ turned out to be notoriously difficult. In a subsequent paper [2], Atiyah and Sutcliffe studied the function At and added two new conjectures (after normalizing At) which imply the original conjecture of Atiyah. They provided compelling numerical evidence of the validity of all three conjectures. The three conjectures can be stated as follows: For all distinct points $P_{1}, \ldots, P_{n}$ of $\mathbb{R}^{3}$ (and all $n \geq 1$ ) we have:

$$
\begin{aligned}
& \operatorname{At}\left(P_{1}, \ldots, P_{n}\right) \neq 0, \\
& \left|\operatorname{At}\left(P_{1}, \ldots, P_{n}\right)\right| \geq \prod_{i<j}\left(2 r_{i j}\right), \quad \text { where } \quad r_{i j}=\left\|\overrightarrow{P_{i} P_{j}}\right\|, \\
& \left|\operatorname{At}\left(P_{1}, \ldots, P_{n}\right)\right|^{n-2} \geq \prod_{k=1}^{n}\left|\operatorname{At}\left(P_{1}, \ldots, P_{k-1}, P_{k+1}, \ldots, P_{n}\right)\right| .
\end{aligned}
$$

From the statement of these conjectures we can see that III $\Longrightarrow$ II $\Longrightarrow$ I. The three conjectures have been very resistant since their inauguration time. The first conjecture was proved by Eastwood and Norbury [5] for the case $n=4$. Other attempts were successful only on special configurations (see [4] and [6]). In this paper, we build on the work of Eastwood and Norbury by presenting a computer aided proof of Conjectures II and III in the case $n=4$ and we also give an elegant factorization of the square of the imaginary part of the Atiyah determinant.

The construction of the determinant is as follows: One starts with $n$ distinct points $P_{1}, \ldots, P_{n}$ $\in \mathbb{R}^{3}$. By considering $P_{j}$ as an observer of the other $n-1$ points we obtain $n-1$ vectors $\vec{P}_{j} P_{1}, \ldots, \vec{P}_{j} P_{j-1},{\overrightarrow{P_{j} P_{j+1}}}_{j}, \ldots,{\overrightarrow{P_{j} P_{n}}}$ in $\mathbb{R}^{3}$. We lift each of these vectors from $\mathbb{R}^{3}$ to $\mathbb{C}^{2}$ using 


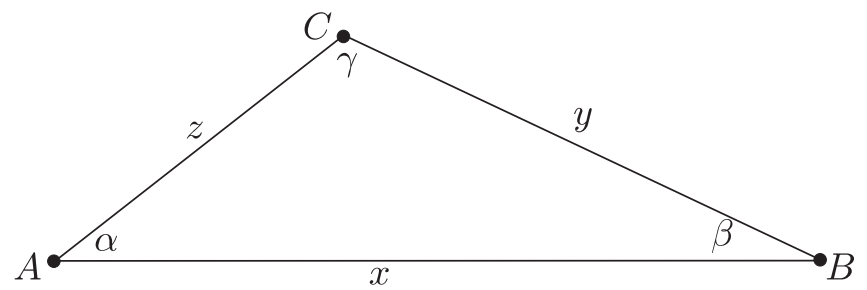

Figure 1. Three points.

the Hopf map $h: \mathbb{C}^{2} \rightarrow \mathbb{R}^{3}$ given by $h(z, w)=\left(\left(|z|^{2}-|w|^{2}\right) / 2, z \bar{w}\right)$ to obtain $n-1$ points of $\mathbb{C}^{2}$. Note that the lifts are not unique and are defined up to phase because $h(\lambda z, \lambda w)=|\lambda|^{2} h(z, w)$. Consequently, our lifts can be considered as points of $\mathbb{C} P^{1}$. Taking the symmetric product of these lifts gives a vector $V_{j}$ in $\mathbb{C} P^{n}$ because $\odot_{n} \mathbb{C} P^{1}=\mathbb{C} P^{n}$. Atiyah's first conjecture was that $\left\{V_{1}, \ldots, V_{n}\right\}$ is a linearly independent set. In other words, the determinant of the matrix having the vector $V_{j}$ as its $j t h$ column is nonzero. This determinant is well-defined up to a phase factor. To get rid of the phase factor ambiguity, we apply the following normalization imposed by Atiyah: If $(z, w)$ is the chosen lift of $\overrightarrow{P_{i} P_{j}}$ and $i<j$, then $(-\bar{w}, \bar{z})$ must be the lift of $\overrightarrow{P_{j} P_{i}}$. After this normalization, this determinant is called the Atiyah determinant and is denoted by At.

It is immediate from the above construction that At is coordinate free and is independent of solid motion. In other words, the determinant function At is invariant under translations and rotations in $\mathbb{R}^{3}$. Furthermore, the Atiyah determinant is built so that it is independent of the order of the points. In other words, if $\left(j_{1}, \ldots, j_{n}\right)$ is a permutation of $(1, \ldots, n)$ then $\operatorname{At}\left(P_{j_{1}}, \ldots, P_{j_{n}}\right)=\operatorname{At}\left(P_{1}, \ldots, P_{n}\right)$. Another property is that At gets conjugated under a plane reflection of the points (see [1]). As a consequence, At must be real-valued if the set of points $\left\{P_{1}, \ldots, P_{n}\right\}$ is symmetric relative to a plane (e.g. if the points are co-planar) since a reflection in the plane leaves the set of points unchanged.

Let us start computing At in the cases $n=2$ and $n=3$. For the case $n=2$, we have two distinct points $A$ and $B$. We can identify $\mathbb{R}^{3}$ with $\mathbb{R} \times \mathbb{C}$ and assume (possibly after a solid motion) that $A$ and $B$ have coordinates $(0,0)$ and $(0, x)$ respectively, where $x>0$ is the distance from $A$ to $B$. By choosing $(\sqrt{x}, \sqrt{x})$ as a lift of $\overrightarrow{A B}$, we are forced to take $(-\sqrt{x}, \sqrt{x})$ as a lift of $\overrightarrow{B A}$. Consequently, Atiyah's determinant is:

$$
\operatorname{At}(A, B)=\left|\begin{array}{cc}
\sqrt{x} & -\sqrt{x} \\
\sqrt{x} & \sqrt{x}
\end{array}\right|=2 x, \quad \text { where } \quad x=\|\overrightarrow{A B}\|
$$

Let us now consider the case $n=3$. Assume (possibly after a solid motion) that $A=$ $(0,0), B=(0, x)$, and $C=\left(0, z e^{I \alpha}\right)$ where $I$ denotes $\sqrt{-1}, y=\|\overrightarrow{B C}\|, z=\|\overrightarrow{A C}\|, x=$ $\|\overrightarrow{A B}\|$ and $\alpha, \beta, \gamma$ are the angles indicated in Fig. 1. When the first point is considered as a vision point we obtain $\overrightarrow{A B}=(0, x), \overrightarrow{A C}=\left(0, z e^{I \alpha}\right)$ whose lifts under the Hopf map $h$ are $(\sqrt{x}, \sqrt{x})$ and $\left(\sqrt{z}, \sqrt{z} e^{-I \alpha}\right)$. And when $B=(0, x)$ is the vision point, we obtain the vectors $\overrightarrow{B A}=(0,-x)$ and $\overrightarrow{B C}=\left(0,-y e^{-I \beta}\right)$ whose lifts are $(-\sqrt{x}, \sqrt{x})$ and $\left(-\sqrt{y}, \sqrt{y} e^{I \beta}\right)$. Similarly, the lifts corresponding to the vision point $C$ are $\left(-\sqrt{z} e^{I \alpha}, \sqrt{z}\right)$ and $\left(-\sqrt{y} e^{-I \beta},-\sqrt{y}\right)$. The symmetric tensor product of the vectors are then $\sqrt{x z}\left(1,1+e^{-I \alpha}, e^{-I \alpha}\right), \sqrt{x y}\left(1,-1-e^{I \beta}, e^{I \beta}\right)$ and $\sqrt{y z}\left(e^{I(\alpha-\beta)}, e^{I \alpha}-e^{-I \beta},-1\right)$, respectively. Consequently, we obtain the Atiyah determinant for three points as

$$
\operatorname{At}(A, B, C)=x y z\left|\begin{array}{ccc}
1 & 1 & e^{I(\alpha-\beta)} \\
1+e^{-I \alpha} & -1-e^{I \beta} & e^{I \alpha}-e^{-I \beta} \\
e^{-I \alpha} & e^{I \beta} & -1
\end{array}\right|
$$




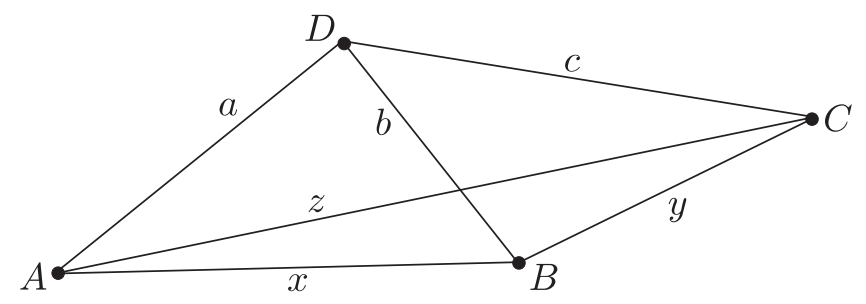

Figure 2. Four points.

This determinant expands to $x y z[6+2(\cos \alpha+\cos \beta+\cos \gamma)]$, which can be written as $x y z[8+$ $\left.8 \sin \frac{\alpha}{2} \sin \frac{\beta}{2} \sin \frac{\gamma}{2}\right]$. Using the identity $\sin \frac{\alpha}{2}=\frac{1}{2} \sqrt{\frac{(a+b-c)(a+c-b)}{b c}}$ and similar identities for $\sin \frac{\beta}{2}$ and $\sin \frac{\gamma}{2}$, we can rewrite the Atiyah determinant for three points as

$$
\operatorname{At}(A, B, C)=8 x y z+d_{3}(x, y, z),
$$

where $d_{3}$ is the polynomial defined by $d_{3}(x, y, z)=(-x+y+z)(x+y-z)(x+y-z)$. From the triangle inequality it follows that $d_{3}(x, y, z)$ is nonnegative, and so Conjecture III is verified for three points. (Note that there is no need to use $\mid$ At $\mid$ because in this case At is real.)

\section{The case of four points}

Given four points $A, B, C, D$ in $\mathbb{R}^{3}$, the vector $u=U(A, B, C, D)$ in $\mathbb{R}^{6}$, called the vector of pair-wise distances, is defined by $u=(a, b, c, x, y, z)$ where $a=\|\overrightarrow{A D}\|, b=\|\overrightarrow{B D}\|, c=\|\overrightarrow{C D}\|$, $x=\|\overrightarrow{A B}\|, y=\|\overrightarrow{B C}\|, z=\|\overrightarrow{A C}\|$ (see Fig. 2). The function $U$, as defined above, maps $\mathbb{R}^{3 \times 4}$ into $\mathbb{R}^{6}$, and it is clear that $U$ is neither injective nor surjective. A vector $u \in \mathbb{R}^{6}$ is said to be geometric if it belongs to the range of $U$. For convenience, we adopt the convention that $\operatorname{At}(A, B, C, D)$ equals 0 when the points $A, B, C, D$ are not distinct, and Conjecture II (for four points) becomes

\section{(II) $|\operatorname{At}(A, B, C, D)| \geq 64 a b c x y z$ for all points $A, B, C, D \in \mathbb{R}^{3}$.}

Atiyah's determinant is designed to be invariant under permutations of the points. Each of the 24 possible permutations of the four points $A, B, C, D$ results in a permutation of the pair-wise distances $a, b, c, x, y, z$. Specifically, if $u=(a, b, c, x, y, z) \in \mathbb{R}^{6}$, then the 24 resultant permutations are

$$
\begin{aligned}
& u_{0}=(a, b, c, x, y, z), \quad u_{1}=(a, x, z, b, y, c), \quad u_{2}=(b, c, a, y, z, x), \quad u_{3}=(x, b, y, a, c, z), \\
& u_{4}=(c, a, b, z, x, y), \quad u_{5}=(z, y, c, x, b, a), \quad u_{6}=(y, z, c, x, a, b), \quad u_{7}=(c, b, a, y, x, z), \\
& u_{8}=(x, y, b, z, c, a), \quad u_{9}=(a, c, b, z, y, x), \quad u_{10}=(z, x, a, y, b, c), \quad u_{11}=(b, a, c, x, z, y), \\
& u_{12}=(z, c, y, a, b, x), \quad u_{13}=(x, z, a, y, c, b), \quad u_{14}=(x, a, z, b, c, y), u_{15}=(y, x, b, z, a, c), \\
& u_{16}=(y, b, x, c, a, z), u_{17}=(y, c, z, b, a, x), u_{18}=(c, y, z, b, x, a), u_{19}=(z, a, x, c, b, y) \text {, } \\
& u_{20}=(b, x, y, a, z, c), u_{21}=(c, z, y, a, x, b), \quad u_{22}=(a, z, x, c, y, b), u_{23}=(b, y, x, c, z, a) .
\end{aligned}
$$

A function $f: \mathbb{R}^{6} \rightarrow \mathbb{R}$ is said to be symmetric if $f(u)=f\left(u_{i}\right)$ for $i=0,1, \ldots, 23$ and is skew-symmetric if $f(u)=(-1)^{i} f\left(u_{i}\right)$ for $i=0,1, \ldots, 23$. The symmetric average of $f$ is the symmetric function av $[f]$ defined by

$$
\operatorname{av}[f](u)=\frac{1}{24} \sum_{i=0}^{23} f\left(u_{i}\right) .
$$


Using Maple, Eastwood and Norbury have found that the real part of $\operatorname{At}(A, B, C, D)$ can be expressed as $\Re \operatorname{At}(A, B, C, D)=d_{4}(u)$, where $d_{4}$ is the homogeneous polynomial of degree 6 given by

$$
d_{4}(u)=60 p_{4}(u)+4 n_{4}(u)+2 z_{4}(u)+12 \mathrm{av}\left[a\left((b+c)^{2}-y^{2}\right) d_{3}(x, y, z)\right],
$$

where $p_{4}(u)=a b c x y z, d_{3}$ is defined in (1.1), $n_{4}(u)=p_{4}(u)-d_{3}(x c, a y, b z)$ and

$$
\begin{aligned}
z_{4}(u)= & a^{2} y^{2}\left(b^{2}+c^{2}+x^{2}+z^{2}\right)+b^{2} z^{2}\left(a^{2}+c^{2}+x^{2}+y^{2}\right)+c^{2} x^{2}\left(a^{2}+b^{2}+y^{2}+z^{2}\right) \\
& -\left(a^{4} y^{2}+a^{2} y^{4}+b^{4} z^{2}+b^{2} z^{4}+c^{4} x^{2}+c^{2} x^{4}\right) \\
& -\left(a^{2} b^{2} x^{2}+a^{2} c^{2} z^{2}+b^{2} c^{2} y^{2}+x^{2} y^{2} z^{2}\right) .
\end{aligned}
$$

Eastwood and Norbury use the notation $144 V^{2}$ in place of $z_{4}(u)$. If $u=U(A, B, C, D)$, the value $z_{4}(u)$ equals $144 V^{2}$, where $V$ denotes the volume of the tetrahedron formed by the points $A, B, C, D$, and it therefore follows that $z_{4}(u) \geq 0$. It would be erroneous to infer from this that the polynomial $z_{4}$ is nonnegative on all of $\mathbb{R}^{6}$; the above statement implies only that $z_{4}$ is nonnegative on geometric vectors.

Having expressed $\Re \operatorname{At}(A, B, C, D)=d_{4}(u)$ as in (2.1), Eastwood and Norbury then invoke the inequalities $z_{4}(u) \geq 0,(b+c)^{2} \geq y^{2}, d_{3}(x, y, z) \geq 0$ and abcxyz $\geq d_{3}(x c, a y, b z)$ (i.e. $\left.n_{4}(u) \geq 0\right)$ to conclude that

$$
|\operatorname{At}(A, B, C, D)| \geq \Re \operatorname{At}(A, B, C, D)=d_{4}(u) \geq 60 p_{4}(u),
$$

which proves Conjecture I and comes close to proving Conjecture II.

Regarding the imaginary part of $\operatorname{At}(A, B, C, D)$, Eastwood and Norbury have shown that its square can be written as $(\Im \operatorname{At}(A, B, C, D))^{2}=F_{4}(u)$, where $F_{4}$ is a symmetric homogeneous polynomial of degree 12 . Whereas $d_{4}$ seems unwilling to be expressed in a simple manner, we have found that $F_{4}$ factors elegantly as

$$
F_{4}=w_{4}^{2} z_{4}
$$

where $w_{4}$ is the skew-symmetric homogeneous polynomial of degree 3 given by

$$
\begin{aligned}
w_{4}(u)= & \left(a^{2}+y^{2}\right)(b-c-x+z)+\left(b^{2}+z^{2}\right)(-a+c+x-y)+\left(c^{2}+x^{2}\right)(a-b+y-z) \\
& +2(c x+y z)(-a+b)+2(a y+x z)(-b+c)+2(b z+x y)(a-c) .
\end{aligned}
$$

Note that since $w_{4}$ is skew-symmetric it follows that $w_{4}^{2}$ is symmetric. As mentioned in the introduction, the imaginary part of $\operatorname{At}(A, B, C, D)$ vanishes whenever the set of four points $\{A, B, C, D\}$ is symmetric about a plane. Interestingly, this property can be derived from the above factorization: Assuming $\{A, B, C, D\}$ is symmetric about a plane, it then follows that $u=U(A, B, C, D)$ is invariant under some odd permutation of the four points $A, B, C, D$. Since $w_{4}$ is skew-symmetric, we must have $w_{4}(u)=0$ and hence $F_{4}(u)=0$.

\section{A linear program related to Conjecture II}

Since $|\operatorname{At}(A, B, C, D)| \geq \Re \operatorname{At}(A, B, C, D)=d_{4}(u)$, in order to prove Conjecture II, it suffices to show that the polynomial $d_{4}$ satisfies

$$
d_{4}(u) \geq 64 p_{4}(u) \quad \text { for all geometric vectors } u \text {. }
$$


If one has in hand a collection $f_{1}, f_{2}, \ldots, f_{k}$ of symmetric homogeneous polynomials of degree 6 which are known to be nonnegative on geometric vectors, then one can 'have a go' at (3.1) by solving the linear program

$$
\begin{array}{ll}
\operatorname{maximize} & \alpha, \\
\text { subject to } & d_{4}=\alpha p_{4}+\sum_{j=1}^{k} \lambda_{j} f_{j}, \quad \text { with } \quad \lambda_{1}, \lambda_{2}, \ldots, \lambda_{k} \geq 0 .
\end{array}
$$

If (3.2) is feasible and if the optimal objective value is $\alpha=64$ (we will see later that $\alpha>64$ is impossible), then we immediately obtain (3.1). The remaining difficulty is that of finding suitable polynomials $\left\{f_{j}\right\}$. One means of generating a large collection of such polynomials, which we now describe, stems from the triangle inequality.

The four points $A, B, C, D$ contain four (possibly degenerate) triangles and each triangle, by means of the triangle inequality, gives rise to three linear polynomials which are nonnegative when $u=(a, b, c, x, y, z)$ is geometric. For example, the triangle $A, B, C$ yields $-x+y+z$, $x-y+z$ and $x+y-z$. In all, there are twelve such linear polynomials which we refer to as triangular variables and use the notation $t=\left(t_{1}, t_{2}, \ldots, t_{12}\right)$, where

$$
\begin{array}{llll}
t_{1}=-a+b+x, & t_{4}=-b+c+y, & t_{7}=-a+c+z, & t_{10}=-x+y+z, \\
t_{2}=a-b+x, & t_{5}=b-c+y, & t_{8}=a-c+z, & t_{11}=x-y+z, \\
t_{3}=a+b-x, & t_{6}=b+c-y, & t_{9}=a+c-z, & t_{12}=x+y-z .
\end{array}
$$

A vector $\alpha \in \mathbb{Z}_{+}^{12}$ is called a multi-index with order $|\alpha|=\alpha_{1}+\alpha_{2}+\cdots+\alpha_{12}$. Employing the standard notation $t^{\alpha}=t_{1}^{\alpha_{1}} t_{2}^{\alpha_{2}} \cdots t_{12}^{\alpha_{12}}$, we see that $t^{\alpha}$ represents a homogeneous polynomial of degree $|\alpha|$ in the variables $(a, b, c, x, y, z)$. Applying the symmetric average, we conclude that av $\left[t^{\alpha}\right]$ represents a symmetric homogeneous polynomial of degree $|\alpha|$ which is nonnegative on geometric vectors. For integers $\ell \geq 0$, we define $\mathbb{T}_{\ell}$ to be the set of all polynomials av $\left[t^{\alpha}\right]$ with $|\alpha|=\ell$ :

$$
\mathbb{T}_{\ell}=\left\{\operatorname{av}\left[t^{\alpha}\right]:|\alpha|=\ell\right\} .
$$

Numerically, we have found that if one chooses $\left\{f_{j}\right\}$ equal to $\mathbb{T}_{6}$, then the linear program (3.2) is feasible and has optimal objective value $\alpha=32$. The formulation (2.1) of Eastwood and Norbury can be understood in the context of (3.2) as the result of including, in addition to $\mathbb{T}_{6}$, the two symmetric polynomials $z_{4}$ and $n_{4}$ which are nonnegative on geometric vectors. Numerically solving (3.2) with $\left\{f_{j}\right\}$ equal to $\left\{z_{4}, n_{4}\right\} \cup \mathbb{T}_{6}$, we have found that the optimal objective value is $\alpha=60$, and (2.1) is indeed an optimal solution of (3.2) as the term av $\left[a\left((b+c)^{2}-y^{2}\right) d_{3}(x, y, z)\right]$ can be written as a nonnegative linear combination of polynomials in $\mathbb{T}_{6}$.

In order to further increase the optimal objective value $\alpha$ in (3.2), we need other symmetric polynomials which are nonnegative on geometric vectors. In pursuit of this, we have identified the following twenty-one geometric vectors $u$ where $d_{4}(u)=64 p_{4}(u)$ (all are obtained as $u=$ $U(A, B, C, D)$ with $A, B, C, D$ collinear or non-distinct):

$$
\begin{array}{lll}
(0,1,4,1,4,4), & (0,4,8,4,7,8), & (0,6,0,6,6,0), \\
(0,1,1,1,2,1), & (0,5,5,5,5,5), & (0,8,8,8,1,8), \\
(0,1,3,1,4,3), & (0,6,3,6,8,3), & (0,6,7,6,3,7), \\
(0,6,6,6,9,6), & (0,1,1,1,0,1), & (0,5,3,5,3,3), \\
(3,3,1,0,2,2), & (9,9,7,0,2,2), & (13,13,7,0,6,6), \\
(19,11,7,8,4,12), & (17,13,4,4,9,13), & (15,8,7,7,1,8), \\
(9,8,1,1,7,8), & (11,9,8,2,1,3), & (17,9,2,8,7,15) .
\end{array}
$$


Both $d_{4}$ and $p_{4}$ vanish on the first fifteen of these vectors (counting horizontally), but are nonzero on the remaining six. In particular, since $d_{4}(9,8,1,1,7,8)=64 p_{4}(9,8,1,1,7,8)=258048>0$, it follows that there are no feasible solutions of (3.2) with $\alpha>64$. On the other hand, if a feasible solution of (3.2) has been obtained with $\alpha=64$, then it follows that $f_{j}$ vanishes on all of the vectors in (3.3), whenever $\lambda_{j}>0$. It has been verified that $z_{4}$ vanishes on all of these vectors, but $n_{4}$ does not. Therefore, the coefficient of $n_{4}$ will be 0 if (3.2) has been solved with $\alpha=64$. We have considered numerous symmetric homogeneous polynomials of degree 6 which vanish on the vectors in (3.3), but only one of these has resulted in an improvement. Let $v_{4}$ denote the skew-symmetric homogeneous polynomial of degree 3 defined by

$$
v_{4}(u)=(b+z-c-x)(c+x-a-y)(a+y-b-z) .
$$

Then $v_{4}$ vanishes on the vectors in (3.3), and numerically solving (3.2) with $\left\{f_{j}\right\}$ equal to $\left\{z_{4}, n_{4}, v_{4}^{2}\right\} \cup \mathbb{T}_{6}$, we have found that the optimal objective value is $\alpha=188 / 3$. Our obtained identity, which has been verified in $\mathrm{Maple}^{1}$, is the following:

$$
d_{4}(u)=\frac{188}{3} p_{4}(u)+\frac{10}{3} z_{4}(u)+\frac{4}{3} n_{4}(u)+\frac{2}{3} v_{4}^{2}(u)+\frac{1}{3} \sum_{|\alpha|=6} \lambda_{\alpha} \operatorname{av}\left[t^{\alpha}\right],
$$

where the six nonzero coefficients $\lambda_{\alpha}$ and corresponding multi-indices $\alpha$ are given by

$\begin{array}{llllll}\alpha & \lambda_{\alpha} & \alpha & \lambda_{\alpha} & \alpha & \lambda_{\alpha} \\ 000,001,010,112 & 6 & 000,001,011,111 & 18 & 000,001,110,102 & 6 \\ 001,001,001,111 & 14 & 001,001,010,111 & 24 & 001,011,100,110 & 24\end{array}$

\section{Proof of Conjecture II for four points}

Let $m_{4}$ be the symmetric homogeneous polynomial of degree 6 defined by $m_{4}=d_{4}-\left(64 p_{4}+\right.$ $\left.4 z_{4}+v_{4}^{2}\right)$, so that

$$
d_{4}=64 p_{4}+4 z_{4}+v_{4}^{2}+m_{4} .
$$

We will show that $m_{4}$ is nonnegative on geometric vectors, but unfortunately, we have been unable to formulate a proof using only polynomials of degree 6 . Rather, we have had to multiply $m_{4}$ by $p_{4}$ and then work with polynomials of degree 12 .

Theorem 1. The product $p_{4} m_{4}$ can be written as a nonnegative linear combination of polynomials in $\mathbb{T}^{12}$.

Proof. Using Maple, we have verified that $64 p_{4} m_{4}$ can be written as

$$
64 p_{4}(u) m_{4}(u)=\sum_{|\alpha|=12} \lambda_{\alpha} \operatorname{av}\left[t^{\alpha}\right],
$$

where the sixty-four nonzero coefficients $\left\{\lambda_{\alpha}\right\}$ are all positive integers as given in the following table:

$\begin{array}{llllll}\alpha & \lambda_{\alpha} & 011,021,201,112 & 6 & 011,111,220,102 & 6 \\ 001,012,211,112 & 12 & 011,021,201,121 & 6 & 011,112,102,210 & 12 \\ 001,012,211,121 & 12 & 011,021,201,211 & 18 & 011,112,120,210 & 39\end{array}$

\footnotetext{
${ }^{1}$ The sources of our codes are available at http://www.emis.de/journals/SIGMA/2014/070/codes.zip.
} 


$\begin{array}{llllll}001,112,112,012 & 12 & 011,021,221,110 & 54 & 011,112,210,210 & 42 \\ 001,121,121,012 & 21 & 011,022,011,121 & 6 & 011,120,011,212 & 27 \\ 002,110,111,212 & 9 & 011,022,111,102 & 84 & 011,120,011,221 & 24 \\ 002,110,111,221 & 9 & 011,022,111,210 & 18 & 011,120,012,112 & 3 \\ 011,011,021,221 & 30 & 011,022,211,110 & 6 & 011,121,021,201 & 24 \\ 011,011,101,222 & 56 & 011,101,021,212 & 54 & 011,121,102,210 & 6 \\ 011,011,110,222 & 48 & 011,102,012,211 & 6 & 011,121,120,210 & 24 \\ 011,011,112,220 & 6 & 011,102,022,111 & 36 & 011,201,012,211 & 3 \\ 011,011,120,212 & 18 & 011,102,112,102 & 18 & 011,201,021,211 & 45 \\ 011,011,122,102 & 57 & 011,102,112,201 & 24 & 012,012,012,111 & 24 \\ 011,011,201,221 & 36 & 011,102,201,121 & 33 & 012,012,102,111 & 8 \\ 011,011,222,011 & 6 & 011,102,210,112 & 75 & 012,111,012,021 & 36 \\ 011,012,102,112 & 120 & 011,102,210,121 & 12 & 011,021,220,210 & 6 \\ 011,012,112,210 & 18 & 011,110,021,221 & 48 & 011,120,012,202 & 21 \\ 011,012,120,121 & 12 & 011,111,012,202 & 21 & 011,120,021,202 & 24 \\ 011,012,211,102 & 84 & 011,111,021,202 & 18 & 011,201,012,202 & 18 \\ 011,012,211,201 & 72 & 011,111,021,220 & 54 & 011,210,021,202 & 3 \\ 011,021,011,122 & 3 & 011,111,022,102 & 75 & 012,012,120,102 & 3 \\ 011,021,112,012 & 69 & 011,111,202,210 & 12 & & \end{array}$

Corollary 1. The polynomial $m_{4}$ is nonnegative on geometric vectors and consequently (3.1) holds, which proves Conjecture II for four points.

Proof. Let $u=U(A, B, C, D)$ be a geometric vector. It follows from (4.2) that $p_{4}(u) m_{4}(u) \geq 0$. If the points $A, B, C, D$ are distinct, then $p_{4}(u)>0$ and hence $m_{4}(u) \geq 0$. On the other hand, if $A, B, C, D$ are not distinct, then they can be approximated by distinct points $A^{\prime}, B^{\prime}, C^{\prime}, D^{\prime}$ and it will then follow from the continuity of $m_{4}$ that $m_{4}(u) \geq 0$.

\section{Proof of Conjecture III for four points}

Let $P_{4}$ denote the symmetric homogeneous polynomial of degree 12 given by

$$
P_{4}(u):=\left(8 x y z+d_{3}(x, y, z)\right)\left(8 a b x+d_{3}(a, b, x)\right)\left(8 a c z+d_{3}(a, c, z)\right)\left(8 b c y+d_{3}(b, c, y)\right),
$$

whereby $\operatorname{At}(A, B, C) \operatorname{At}(A, B, D) \operatorname{At}(A, C, D) \operatorname{At}(B, C, D)=P_{4}(u)$ when $u=U(A, B, C, D)$. Since $|\operatorname{At}(A, B, C, D)|^{2} \geq(\Re \operatorname{At}(A, B, C, D))^{2}=d_{4}^{2}(u)$, in order to prove Conjecture III, it suffices to show that

$$
d_{4}^{2}(u) \geq P_{4}(u) \text { for all geometric vectors } u \text {. }
$$

Recall from (4.1) that $d_{4}$ has been written as $d_{4}=64 p_{4}+m_{4}+\left(4 z_{4}+v_{4}^{2}\right)$, so it follows that

$$
\begin{aligned}
d_{4}^{2} & =\left(4 z_{4}+v_{4}^{2}\right) d_{4}+\left(64 p_{4}+m_{4}\right) d_{4} \\
& =\left(4 z_{4}+v_{4}^{2}\right) d_{4}+\left(64 p_{4}+m_{4}\right)^{2}+\left(64 p_{4}+m_{4}\right)\left(4 z_{4}+v_{4}^{2}\right) \\
& =\left(4 z_{4}+v_{4}^{2}\right)\left(d_{4}+32 p_{4}+m_{4}\right)+\left(64 p_{4}+m_{4}\right)^{2}+32 p_{4}\left(4 z_{4}+v_{4}^{2}\right) .
\end{aligned}
$$

With $M_{4}$ denoting the symmetric homogeneous polynomial of degree 12 defined by $M_{4}=$ $\left(64 p_{4}+m_{4}\right)^{2}+32 p_{4}\left(4 z_{4}+v_{4}^{2}\right)-P_{4}$, we then have

$$
d_{4}^{2}=P_{4}+\left(4 z_{4}+v_{4}^{2}\right)\left(d_{4}+32 p_{4}+m_{4}\right)+M_{4} .
$$


Theorem 2. The polynomial $M_{4}$ is nonnegative on geometric vectors, and consequently (5.1) holds, which proves Conjecture III for four points.

Proof. Using Maple, we have verified that $128 M_{4}$ can be written as

$$
128 M_{4}(u)=\left(4 z_{4}(u)+v_{4}^{2}(u)\right) \sum_{|\alpha|=6} \mu_{\alpha} \operatorname{av}\left[t^{\alpha}\right]+\sum_{|\alpha|=12} \nu_{\alpha} \operatorname{av}\left[t^{\alpha}\right]
$$

where the coefficients $\left\{\mu_{\alpha}\right\}$ and $\left\{\nu_{\alpha}\right\}$ are nonnegative integers: The 6 nonzero coefficients $\mu_{\alpha}$ and corresponding monomials $\alpha$ are given in the following table:

$\begin{array}{llllll}000,001,111,110 & 1236 & 000,101,101,101 & 3594 & 001,010,011,011 & 300 \\ 000,100,101,111 & 60 & 000,101,110,101 & 114 & 001,011,101,001 & 1014\end{array}$

The 114 nonzero coefficients $\nu_{\alpha}$ and corresponding monomials $\alpha$ are given in the following table:

\begin{tabular}{|c|c|c|c|c|c|}
\hline $000,112,121,112$ & 2019 & $011,120,021,112$ & 2184 & $001,120,202,121$ & 76 \\
\hline $001,012,121,112$ & 369 & $011,121,021,201$ & 228 & $001,121,201,220$ & 6 \\
\hline $01,012,211,121$ & 138 & $011,121,210,210$ & 72 & $001,121,220,021$ & 3174 \\
\hline $01,012,211,211$ & 666 & $011,201,021,211$ & 936 & $001,121,220,120$ & 1266 \\
\hline $1,021,211,121$ & 3087 & $012,012,012,111$ & 3072 & $001,122,210,120$ & 1428 \\
\hline $1,022,111,112$ & 3009 & $012,111,021,201$ & 1308 & $001,201,220,112$ & 822 \\
\hline $1,022,111,121$ & 1074 & $012,111,210,012$ & 5374 & $001,210,221,021$ & 612 \\
\hline $1,022,112,111$ & 42 & $000,012,111,222$ & 60 & $001,211,220,012$ & 300 \\
\hline $1,022,211,111$ & 12114 & $000,012,112,212$ & 1776 & $001,211,220,120$ & 3072 \\
\hline $1,101,211,122$ & 240 & $001,001,112,222$ & 138 & $002,002,112,112$ & 1536 \\
\hline $01,111,122,102$ & 4056 & $001,001,122,212$ & 1398 & $002,011,221,021$ & 1176 \\
\hline $01,111,210,221$ & 444 & $001,002,122,112$ & 3072 & $002,012,110,212$ & 1662 \\
\hline $1,120,121,112$ & 144 & $001,002,211,122$ & 1236 & $002,012,210,121$ & 5136 \\
\hline $1,121,210,112$ & 714 & $001,010,112,222$ & 768 & $002,012,210,211$ & 762 \\
\hline $1,121,210,211$ & 1146 & $001,010,122,212$ & 384 & $002,012,211,021$ & 2178 \\
\hline $1,211,220,111$ & 1866 & $001,010,212,122$ & 384 & $002,022,110,112$ & 1188 \\
\hline $02,011,111,122$ & 2808 & $001,011,102,222$ & 2568 & $002,022,110,121$ & 150 \\
\hline $02,011,211,121$ & 3207 & $001,011,222,012$ & 1224 & $002,022,211,011$ & 2178 \\
\hline $02,012,111,112$ & 3654 & $001,012,110,222$ & 2634 & $002,101,101,222$ & 1245 \\
\hline $002,012,111,121$ & 3252 & $001,012,120,212$ & 66 & $002,101,122,102$ & 246 \\
\hline $002,021,111,112$ & 276 & $001,012,122,102$ & 822 & $002,101,221,012$ & 528 \\
\hline $002,022,111,111$ & 720 & $001,021,112,220$ & 840 & $002,102,121,102$ & 222 \\
\hline $02,111,212,011$ & 516 & $001,022,120,112$ & 4542 & $002,110,122,102$ & 7548 \\
\hline $002,112,210,111$ & 1206 & $001,022,211,120$ & 2928 & $002,121,210,012$ & 1074 \\
\hline $02,112,211,011$ & 1662 & $001,022,220,111$ & 6546 & $002,122,210,011$ & 60 \\
\hline $011,011,012,212$ & 10110 & $001,101,102,222$ & 2898 & $011,012,120,220$ & 3072 \\
\hline $011,011,021,212$ & 1164 & $001,101,201,222$ & 804 & $011,021,210,220$ & 342 \\
\hline $011,011,201,212$ & 5472 & $001,101,220,122$ & 1398 & $011,021,220,120$ & 4668 \\
\hline $1,012,012,211$ & 342 & $001,102,110,222$ & 690 & $011,021,220,210$ & 4608 \\
\hline
\end{tabular}




$\begin{array}{llllll}011,012,112,201 & 2178 & 001,102,122,102 & 1770 & 011,022,120,102 & 768 \\ 011,012,122,101 & 192 & 001,102,202,112 & 5532 & 011,022,120,210 & 1536 \\ 011,021,211,120 & 5472 & 001,102,202,121 & 612 & 011,022,201,120 & 1890 \\ 011,022,111,120 & 2376 & 001,102,210,212 & 390 & 011,022,210,102 & 1152 \\ 011,022,121,110 & 696 & 001,102,221,102 & 2634 & 011,022,210,210 & 6648 \\ 011,101,022,112 & 2628 & 001,110,201,222 & 192 & 011,120,022,102 & 10752 \\ 011,102,201,112 & 372 & 001,112,220,120 & 600 & 011,210,012,202 & 168 \\ 011,110,012,122 & 3558 & 001,112,221,002 & 60 & 011,210,120,202 & 522 \\ 011,112,021,201 & 948 & 001,120,201,221 & 774 & 012,120,012,201 & 4440\end{array}$

It now follows from (5.3) that $M_{4}$ is nonnegative on geometric vectors and we obtain (5.1) as a consequence of (5.2).

\section{References}

[1] Atiyah M., The geometry of classical particles, in Surveys in Differential Geometry, Surv. Differ. Geom., VII, Int. Press, Somerville, MA, 2000, 1-15.

[2] Atiyah M., Sutcliffe P., The geometry of point particles, Proc. Roy. Soc. London Ser. A 458 (2002), 10891115, hep-th/0105179.

[3] Berry M.V., Robbins J.M., Indistinguishability for quantum particles: spin, statistics and the geometric phase, Proc. Roy. Soc. London Ser. A 453 (1997), 1771-1790.

[4] Doković D.Ž., Verification of Atiyah's conjecture for some nonplanar configurations with dihedral symmetry, Publ. Inst. Math. (Beograd) (N.S.) 72 (2002), 23-28.

[5] Eastwood M., Norbury P., A proof of Atiyah's conjecture on configurations of four points in Euclidean three-space, Geom. Topol. 5 (2001), 885-893, math.MG/0109161.

[6] Mazur M., Petrenko B.V., On the conjectures of Atiyah and Sutcliffe, Geom. Dedicata 158 (2012), 329-342, arXiv:1102.4662. 